\title{
Severe skin disease in lupus associated with hemophagocytic lymphohistiocytosis: case reports and review of the literature
}

\author{
Christina S. Thornton ${ }^{1}\left(\mathbb{D}\right.$, Parham Minoo ${ }^{2}$, Michelle Schneider ${ }^{2}$ and Aurore Fifi-Mah ${ }^{3,4^{*}}$
}

\begin{abstract}
Background: Hemophagocytic lymphohistiocytosis $(H L H)$ is a severe clinical entity associated with high mortality in the adult population. HLH has been associated with infections, malignancy and autoimmune conditions such as Systemic Lupus Erythematosus (SLE), however this is often in the context of a disease flare. Currently, there are limited reports of inaugural SLE manifesting as HLH with a lack of consensus on treatment and management of these patients.

Case presentation: Here, we present two rare case reports of severe cutaneous manifestation of lupus associated with HLH. Both patients presented with sinister clinical courses with primarily rheumatologic complaints including malaise, arthralgia, and myalgia with biochemical abnormalities. Both patients were diagnosed with HLH as a result of first presentation from cutaneous lupus. A comprehensive literature review using the PubMed database with cases comprising keywords of HLH and SLE up to September 2017 was conducted, with an emphasis on inaugural cutaneous SLE cases.
\end{abstract}

Conclusions: Ultimately, we highlight that a keen clinical acumen is required as misdiagnosis may lead to insufficient treatment with adverse clinical outcomes with the unique presentation of HLH from inaugural cases of SLE.

\section{Background}

Hemophagocytic lymphohistiocytosis (HLH) is a constellation of symptoms caused by dysregulated hyperinflammation and cytokine storm, resulting in a life-threatening syndrome. HLH is classified into primary (familial) and secondary etiologies (infection, autoimmune conditions, drugs and malignancy) [1]. Often, HLH is associated with pediatric rheumatic conditions, however there is a growing body of literature reporting HLH in the older population.

Clinically and biochemically, the hallmark features include hepatosplenomegaly, fever, hyperferritinemia, hypofibrinogenemia, hypertriglyceridemia and pancytopenia [2]. While the true incidence of HLH is unknown, the mortality if left untreated is high and often patients succumb in days to months from multi-organ failure.

\footnotetext{
* Correspondence: Aurore.FifiMah@albertahealthservices.ca; aurore.fifimah@albertahealthservices.ca

${ }^{3}$ Division of Rheumatology, Department of Medicine, University of Calgary, Calgary, Alberta, Canada

${ }^{4}$ Rheumatology Outpatient Clinic, South Health Campus, 4448 Front Street SE, Calgary, Alberta T2M 1M4, Canada

Full list of author information is available at the end of the article
}

However, with rapid identification of HLH and initiation of treatment, the survival rate approaches 50\% [3, 4].

Given the main barrier in treatment is delay in diagnosis; HLH requires keen clinical acuity in order to attenuate complications from the sequela of disease. While there have been several reports in the literature of HLH in adulthood associated with autoimmune conditions such as systemic lupus erythematosus (SLE), often these have been associated with disease flares. We present here two cases of cutaneous lupus as the initial manifestation of HLH, a rare but clinically relevant entity. Accurate diagnosis is critical as the therapeutic approach may differ depending on the severity of HLH manifestation $[5,6]$.

\section{Case presentation}

A 73-year-old Caucasian male presented to our acute care tertiary hospital with a several day history of rash that initially started on the scalp and was felt to be due to sunburn from outdoor exposure, but subsequently spread over the torso and arms with associated blistering. He also 
began to develop increasing fatigue and malaise, which prompted him to seek medical attention. His past medical history was significant only for hypertension and osteoarthritis. He denied any medications but did acknowledge alcohol substance use disorder. Remainder of review of systems was otherwise unremarkable.

On admission, he was febrile at $38.9^{\circ} \mathrm{C}$, heart rate was 110 beats/min, blood pressure was $105 / 82$ and respiratory rate was 18 breaths/min. Physical examination was significant for skin findings including multiple flaccid bullae on an erythematous base with serosanguinous fluid diffusely over the torso, back and arms. A thick confluent plaque over the scalp was also noted. Palpable purpura at the lower extremities was present with petechiae to the fingers and toes. There was no mucosal involvement. The remainder of physical examination including precordium, respiratory and abdomen were within normal limits.

Initial laboratory investigations revealed pancytopenia (hemoglobin: $105 \mathrm{~g} / \mathrm{L}$; platelets: $53 \times 10^{9} / \mathrm{L}$, white blood cell: $\left.3.3 \times 10^{9} / \mathrm{L},\right)$, CRP of $19.1 \mathrm{mg} / \mathrm{L}(0-8 \mathrm{mg} / \mathrm{L})$ and ESR of $28 \mathrm{~mm}(0-10 \mathrm{~mm})$. Haptoglobin was low at $0.09 \mathrm{~g} / \mathrm{L} \quad(0.3-2.0 \mathrm{~g} / \mathrm{L})$, suggesting an element of hemolysis. Albumin was low at $23 \mathrm{~g} / \mathrm{L}(33-48 \mathrm{~g} / \mathrm{L})$ lactate dehydrogenase was increased at 349 U/L (100-235 $\mathrm{U} / \mathrm{L})$, as well as alanine aminotransferase at $141 \mathrm{U} / \mathrm{L}$ (1-40 U/L) and gamma glutamyl-transferase at $201 \mathrm{U} / \mathrm{L}$ (11-63 U/L). Ferritin was profoundly elevated at > $8000 \mu \mathrm{g} / \mathrm{L}(13-150 \mu \mathrm{g} / \mathrm{L})$. Fibrinogen and D-dimer were within normal limits. Triglycerides were mildly elevated at $2.04 \mathrm{mmol} / \mathrm{L}(0.0-1.70 \mathrm{mmol} / \mathrm{L})$. An initial immunological work-up showed an ANA titre of 1:80 with a homogenous and speckled pattern. ENA revealed positive Smith and RNP antibody. C3 and C4 were both depressed at $0.32 \mathrm{~g} / \mathrm{L}(0.6-1.6 \mathrm{~g} / \mathrm{L})$ and $0.04 \mathrm{~g} / \mathrm{L}(0.1-0.4$ $\mathrm{g} / \mathrm{L})$, respectively. Of note, atypical ANCA was observed on indirect immunofluorescence but MPO and PR3 ANCA by ELISA were negative. Soluble IL-2R by ALBIA (addressable laser bead immunoassay methodology) was high. Abdominal ultrasound demonstrated heavy hepatic steatosis but no evidence of hepatosplenomegaly. Chest X-ray and echocardiogram were within normal parameters.

Due to the new onset of pancytopenia, a bone marrow biopsy was performed revealing a hypercellular marrow with granulocyte hyperplasia and the presence of hemophagocytosis; suggestive of HLH (Fig. 1). Skin biopsies from the torso showed full-thickness epidermal necrosis with subepidermal blister formation and absence of hemophagocytosis. A second skin biopsy done from the purpuric right fifth toe showed ulceration with focal epidermal and fat necrosis and dermal hemorrhage suggestive of ischemic changes.

$\mathrm{He}$ also began to clinically deteriorate with refractory hypotension and tachycardia. At this time, he was suspected to have new onset acute cutaneous SLE presenting with bullous lesions with secondary HLH. Therefore, he was treated with methylprednisolone 1000 mg IV daily for a three-day pulse course and then was switched to prednisone $60 \mathrm{mg}$ daily. He was also started on hydroxychloroquine $400 \mathrm{mg}$ daily. At time of discharge, his blood counts had slightly improved and fevers had dissipated with hemodynamic stability. One month after discharge, he was seen in the outpatient rheumatology clinic with resolution of the skin rash and improving blood counts (hemoglobin $112 \mathrm{~g} / \mathrm{L}$, platelets and white blood cell count normal) and ferritin of $1065 \mu \mathrm{g} / \mathrm{L}$.

\section{Case presentation}

A 43-year-old African-Canadian male presented with a 6-week history of weight loss of 20 pounds, fatigue, persistent low-grade fever and a prominent malar rash and extensive diffuse desquamative rash on most of the body. On further history, he noted arthralgia for the preceding 20 years and a similar intermittent rash. Past medical history was non-contributory and he denied any medication use. Review of systems was otherwise unremarkable.

Initial laboratory investigations showed a complete blood count that was significant for hemoglobin $84 \mathrm{~g} / \mathrm{L}$, platelets of $80 \times 10^{9} / \mathrm{L}$, alanine aminotransferase at 145 $\mathrm{U} / \mathrm{L}$, fibrinogen of $110 \mathrm{mg} / \mathrm{dL}$ and creatinine of 145 $\mu \mathrm{mmol} / \mathrm{L}$. He was subsequently admitted under the general internal medicine team for management of acute kidney injury.

Further bloodwork showed a positive ANA of 1:640 with a speckled morphology, anti-Ds DNA intermediate at $9 \mathrm{kIU} / \mathrm{L}$ and ENA with moderately positive U1-RNP, high positive Smith antigen, low positive Scl-70 and SS-A/Ro 60 moderately positive. Decreased C3 of $0.15 \mathrm{~g} /$ $\mathrm{L}, \mathrm{C} 4$ of $0.02 \mathrm{~g} / \mathrm{L}$, and ferritin of $2989 \mu \mathrm{g} / \mathrm{L}$ were also observed. He was treated with methylprednisolone 1000 mg IV daily for a three-day pulse course. The clinical course deteriorated with anuric renal failure requiring hemodialysis and hypoxic respiratory failure secondary to pneumonia precipitating ICU admission and intubation. Bloodwork done at that time showed ferritin level of greater than $100,000 \mu \mathrm{g} / \mathrm{L}$, decreased fibrinogen of $1.5 \mathrm{~g} / \mathrm{L}$ and elevated triglycerides of $5.5 \mathrm{mmol} / \mathrm{L}$; in keeping with development of HLH secondary to SLE. Renal biopsy in the context of anuria showed lupus nephritis, class I with acute tubular injury. The patient required multiple courses of methylprednisolone IV then switched to oral prednisone, anakinra, cyclophosphamide IV and hydroxychloroquine. Eventually, his clinical course improved with ability to be extubated and subsequently discharged. At time of discharge, his blood counts had improved and the cutaneous rash had dissipated. He continues to be followed in the outpatient 


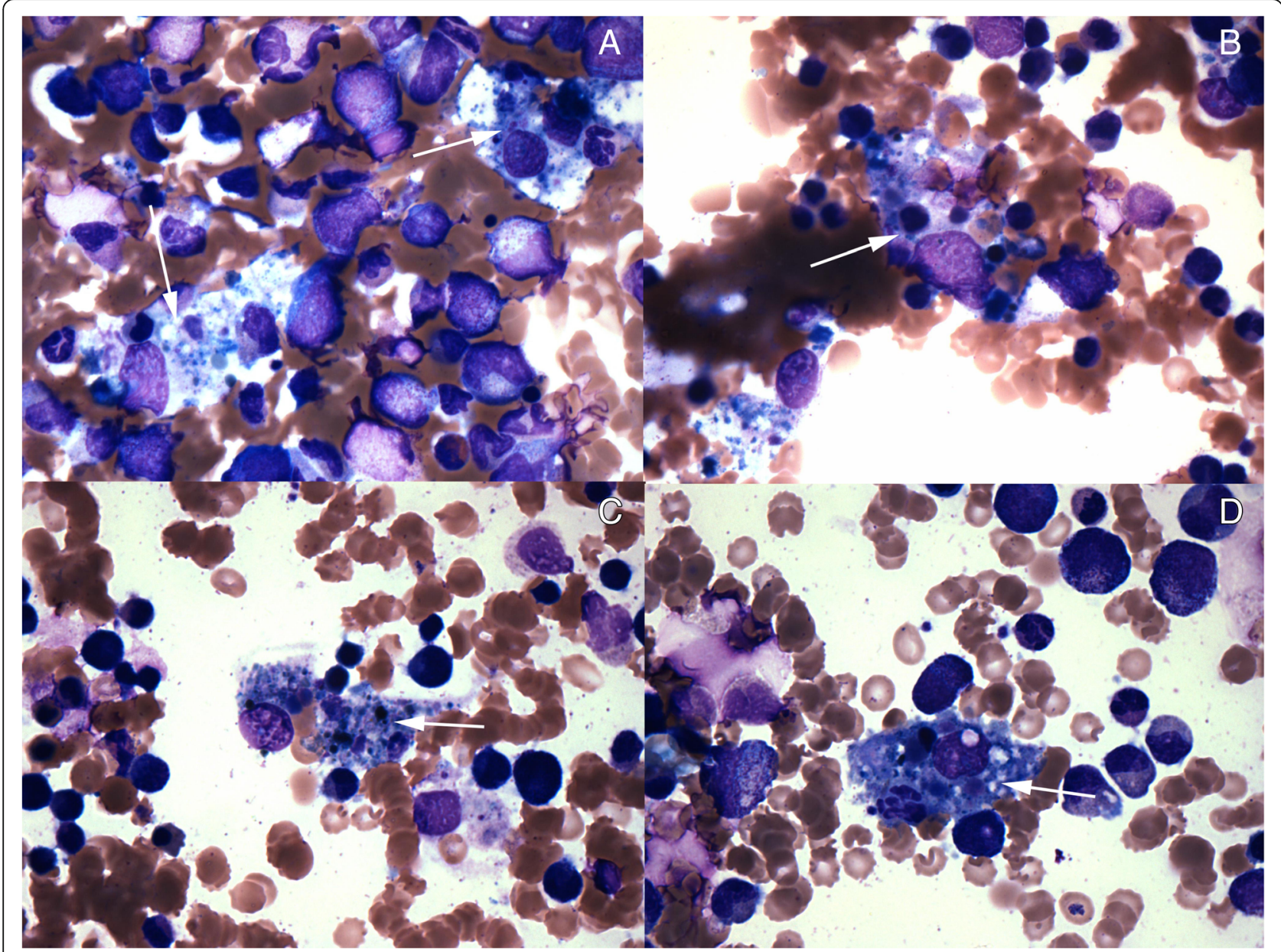

Fig. 1 Bone Marrow Biopsy of HLH (a-d). The marrow showed trilineage hematopoiesis with relative granulocyte hyperplasia (M:E ratio 6.2:1). Erythroid and granulocyte precursors showed orderly maturation with no evidence of dyspoiesis or increased blasts. Megakarycoytes were adequately present and show normal morphology. Lymphocytes and plasma cells were unremarkable. Macrophages were mildly increased with evidence of hemophagocytosis and show frequent forms with ingested mature red cells, erythroid precursors, occasional leukocytes and platelets. Arrows indicate sections associated with hemophagocytosis

rheumatology setting and disease has been quiescent on mycophenolate mofetil $1 \mathrm{~g}$ twice daily, anakinra $100 \mathrm{mg}$ subcutaneous daily, and hydroxychloroquine $400 \mathrm{mg}$ daily. This case represents a delayed diagnosis of SLE progressing to severe skin manifestation of the disease complicated with secondary HLH (Table 1).

\section{Discussion and conclusions}

HLH is a rare but devastating clinical entity and has been associated with several rheumatologic disorders including adult Still's disease, sarcoidosis, systemic sclerosis and Sjogren's syndrome [7]. An extensive literature review was completed for articles published up to September 2017 based on a bibliographic search in the PubMed database using the keywords "Hemophagocytic lymphohistiocytosis" and "Systemic lupus erythematosus" with inclusion criteria of articles focusing on cutaneous manifestation. Case reports involving new diagnosis of SLE as the onset of HLH is limited [8-12]. In the cases presented here, case 1 and 2's clinical onset of HLH did coincide with the new onset of SLE and does fulfill the SLICC criteria for SLE. Amongst all cases of SLE and HLH, including rheumatologic flares, the incidence of HLH is approximately $0.9-4.6 \%$ [13]. As has been described by several groups, the difficulty in making the diagnosis of HLH in new onset SLE arise from the overlap of many symptoms, making the use of the HLH-2004 criteria critical for acute and timely diagnosis. Several other parameters have been used to tease out the elusive diagnosis, however with mixed consensus in the literature. Hyperferritinemia has been cited as the best parameter to distinguish between active SLE flare and HLH-associated SLE with a sensitivity and specificity of nearly $100 \%$ [1]. However, hyperferritinemia in the HLH-94 study indicated that in the pediatric population, ferritin level $>500 \mathrm{mcg} / \mathrm{L}$ was $100 \%$ sensitive for HLH but less specific; whereas ferritin $>10,000 \mathrm{mcg} / \mathrm{L}$ was $90 \%$ 
Table 1 Diagnostic Criteria of HLH as defined by the HLH-2004 Criteria

\begin{tabular}{lll}
\hline HLH Criteria & Case & Case \\
& 1 & 2 \\
\hline Fever $\geq 38.5^{\circ} \mathrm{C}$ & + & + \\
Splenomegaly & - & - \\
Hemoglobin $<9 \mathrm{~g} / \mathrm{dL}^{\text {a }}$ & - & + \\
Platelets $<100,000$ microL $^{\mathrm{a}}$ & + & + \\
Absolute Neutrophil Count $<1000 / \mathrm{microl}^{\mathrm{a}}$ & - & - \\
Hypertriglyceridemia: Fasting Triglycerides $>$ & + & + \\
265 mg/dL & & \\
Hypofibrinogenemia: Fibrinogen $<150 \mathrm{mg} / \mathrm{dL}$ & - & + \\
Hemophagocytosis in bone marrow, spleen, & + & - \\
lymph node or liver & & \\
Low or absent NK cell activity & $\mathrm{n} / \mathrm{a}$ & $\mathrm{n} / \mathrm{a}$ \\
$\begin{array}{l}\text { Ferritin }>500 \mathrm{ng} / \mathrm{mL} \\
\text { Elevated soluble CD25 (soluble } \mathrm{IL}-2 \text { receptor alpha) two }\end{array}$ & + & + \\
standard deviations above age-adjusted laboratory- & & \\
specific norms & & \\
\hline
\end{tabular}

${ }^{a}$ Peripheral blood cytopenia as one criteria

sensitive and 96\% specific for HLH [1]. In the adult population, the correlation between hyperferritinemia and HLH is less clear, particularly with the presence of an overlapping autoimmune condition such as SLE. One study evaluated HLH-associated SLE with solely active SLE and concluded a high ferritin level often points towards the former [7].

More recently, a group from France has published the "HScore", a well-validated scoring system for the diagnosis of HLH [1]. The scoring system incorporates parameters including organomegaly, ferritin, ALT, degree of cytopenia, fibrinogen, fever, and hemophagocytosis with a HScore $>250$ conferring $99 \%$ probability of HLH and a score $<90$ at $<1 \%$ probability. Interestingly, for case 1 the HScore was calculated as 245 (conferring 99.1\% probability of HLH) and case 2 as 201 (conferring 88.8\% probability for HLH). In the future, the HScore may be a useful clinical parameter to help tease out active SLE from HLH-associated SLE.

The two cases here highlight an extremely rare entity within HLH-associated SLE with severe cutaneous manifestation at the time of diagnosis. A case report from Japan also observed this entity with erythematous plaques as the initial manifestation of HLH in a patient with newly diagnosed lupus [14]. Furthermore, HLH can present with skin manifestation but (in) the absence of antibodies can eliminate an associated autoimmune process.

In patients with known SLE, the parameters to gauge flare from $\mathrm{HLH}$ is even more difficult. One study assessed patients with known autoimmune conditions (including SLE) and performed skin biopsies on three patients during acute flare, of which all were found to have hemophagocytosis [15]. While this may be an important additional clue to help tease apart the two conditions, hemophagocytosis is a non-specific finding and has been associated with self-limiting bone marrow infections in the context of activated macrophages. A laboratory study assessed hemophagocytosis by comparing bone marrow aspirates in patients with known HLH compared to random control bone marrows [16]. The sensitivity of hemophagocytosis in HLH was $83 \%$ with a specificity of only $60 \%$, suggesting rare hemophagocytes may be seen in normal bone marrow. The authors suggest a rise of hemophagocytosis count threshold to increase to $0.05-0.13 \%$ to account for rare 'normal' markers would increase specificity to $100 \%$, which may also aid in teasing out HLH-associated SLE from SLE flare.

Amongst all currently available immunologic studies available, sIL-2R appears to correlate best with disease activity. One study assessed patients with lymphoma-associated HLH compared to non-lymphoma cases and found that the former had a much higher sIL-2R to ferritin ratio (8.56 vs. 0.66$)$ [17]. These ratios have not been assessed in autoimmune associated HLH, specifically SLE, and may provide a promising avenue to better discern this diagnosis.

Classically, treatment of primary HLH is directed towards use of the HLH-2004 protocol including etoposide, dexamethasone, cyclosporine, consideration of intrathecal methotrexate and finally with hematopoietic stem cell transplantation [18]. Treatment of secondary HLH is less clear, but ultimately relies on treatment of the primary auto-immune disease. In the two cases presented, SLE was the process associated with HLH and corticosteroids were rapidly initiated, in keeping with previously described cases [19]. Because of the high mortality risk of HLH, the absence of rapid improvement of symptoms, even in the context of non-severe manifestations of SLE, requires aggressive immunosuppression with cyclophosphamide and frequently the addition of biologics such as Anakinra (Il1 inhibitor), infliximab (TNF inhibitor) [20] and alemtuzumab (CD52 inhibitor) [6, 21, 22].

Based on the cases and literature review presented here, we suggest considering HLH in the differential diagnosis with adult patients presenting with new onset, severe cutaneous manifestation of lupus and to consider appropriate investigations and prompt treatment.

\section{Abbreviations}

ANA: Anti-nuclear antibody; ANCA: Anti-neutrophil cytoplasmic antibody; CRP: C-reactive protein; ENA: Extractable nuclear antigen;

HLH: Hemophagocytic lymphohistiocytosis; IL-2: Interleukin-2; MPOANCA: Myeloperoxidase-antineutrophil cytoplasmic antibody; SLE: Systemic lupus erythematosus 


\section{Acknowledgements}

The authors gratefully acknowledge the contributions of the two patients involved in the case report.

\section{Funding}

Not applicable.

\section{Availability of data and materials}

Data are contained within the manuscript.

\section{Authors' contributions}

CST and AFM treated the patient and designed the study. CST, PM, MH and AFM collected and analyzed the data and wrote the article. All authors have read and approved the final manuscript.

\section{Ethics approval and consent to participate}

All procedures were part of the standard medical care, and the need for ethics approval and consent to participate was waived.

\section{Consent for publication}

Written informed consent was obtained from the patient for publication of this case report and any accompanying images. A copy of the consent form is available for review by the Editor of this journal.

\section{Competing interests}

The authors declare that they have no competing interests.

\section{Publisher's Note}

Springer Nature remains neutral with regard to jurisdictional claims in published maps and institutional affiliations.

\section{Author details}

'Department of Medicine, University of Calgary, Calgary, Alberta, Canada. ${ }^{2}$ Department of Pathology and Laboratory Medicine, University of Calgary, Calgary, Alberta, Canada. 'Division of Rheumatology, Department of Medicine, University of Calgary, Calgary, Alberta, Canada. ${ }^{4}$ Rheumatology Outpatient Clinic, South Health Campus, 4448 Front Street SE, Calgary, Alberta T2M 1M4, Canada.

Received: 25 September 2018 Accepted: 25 January 2019

Published online: 08 February 2019

\section{References}

1. Vilaiyuk S, Sirachainan N, Wanitkun S, Pirojsakul K, Vaewpanich J. Recurrent macrophage activation syndrome as the primary manifestation in systemic lupus erythematosus and the benefit of serial ferritin measurements: a casebased review. Clin Rheumatol. 2013;32:899-904.

2. Kumakura S, Ishikura H, Munemasa S, Adachi T, Murakawa Y, Kobayashi S. Adult onset Still's disease associated hemophagocytosis. J Rheumatol. 1997; 24:1645-8.

3. Henter Jl, Horne A, Arico M, et al. HLH-2004: diagnostic and therapeutic guidelines for hemophagocytic lymphohistiocytosis. Pediatr Blood Cancer. 2007:48:124-31.

4. Z Zhang JR, Liang XL, Jin R, Lu G. HLH-2004 protocol: diagnostic and therapeutic guidelines for childhood hemophagocytic lymphohistiocytosis. Zhongguo Dang Dai Er Ke Za Zhi. 2013;15:686-8.

5. Wohlfarth $\mathrm{P}$, Agis $\mathrm{H}$, Gualdoni $\mathrm{GA}$, et al. Interleukin 1 receptor antagonis Anakinra, intravenous immunoglobulin, and corticosteroids in the Management of Critically ill Adult Patients with Hemophagocytic Lymphohistiocytosis. J Intensive Care Med 2017: 885066617711386.

6. Grom AA, Horne A, De Benedetti F. Macrophage activation syndrome in the era of biologic therapy. Nat Rev Rheumatol. 2016;12:259-68

7. Trottestam $\mathrm{H}$, Horne A, Arico M, et al. Chemoimmunotherapy for hemophagocytic lymphohistiocytosis: long-term results of the HLH-94 treatment protocol. Blood. 2011;118:4577-84.

8. Dhote R, Simon J, Papo T, et al. Reactive hemophagocytic syndrome in adult systemic disease: report of twenty-six cases and literature review. Arthritis Rheum. 2003:49:633-9.

9. Fukaya S, Yasuda S, Hashimoto T, et al. Clinical features of haemophagocytic syndrome in patients with systemic autoimmune diseases: analysis of 30 cases. Rheumatology (Oxford). 2008;47:1686-91.
10. Hot A, Toh ML, Coppere B, et al. Reactive hemophagocytic syndrome in adult-onset still disease: clinical features and long-term outcome: a casecontrol study of 8 patients. Medicine (Baltimore). 2010;89:37-46.

11. Otrock ZK, Eby CS. Clinical characteristics, prognostic factors, and outcomes of adult patients with hemophagocytic lymphohistiocytosis. Am J Hematol. 2015;90:220-4.

12. Wong KF, Hui PK, Chan JK, Chan YW, Ha SY. The acute lupus hemophagocytic syndrome. Ann Intern Med. 1991;114:387-90.

13. Gavand PE, Serio I, Larroche C, Costedoat-Chalumeau N, Lavigne C, Mouthon L, Artifoni M, Dossier A, Bloch-Queyrat C, Arnaud L, Amoura Z, Martin T. Association syndrome lymphohystiocytaire $(H L H)$ et lupus systémique : étude multicentrique française de 103 épisodes chez 89 patients adultes. La Revue de Médecine Interne. 2016:37:A122-A3.

14. Fardet L, Galicier L, Lambotte O, et al. Development and validation of the HScore, a score for the diagnosis of reactive hemophagocytic syndrome. Arthritis Rheumatol. 2014;66:2613-20.

15. Tochihara M, Kasai M, Katsumata $Y$, et al. Erythematosus plaques with macrophage infiltration as an initial manifestation of macrophage activation syndrome in a patient with systemic lupus erythematosus. Mod Rheumatol. 2016:26:956-7.

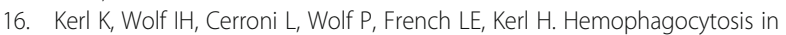
cutaneous autoimmune disease. Am J Dermatopathol. 2015:37:539-43.

17. Goel S, Polski JM, Imran H. Sensitivity and specificity of bone marrow hemophagocytosis in hemophagocytic lymphohistiocytosis. Ann Clin Lab Sci. 2012;42:21-5.

18. Henter II, Horne AC, Arico M, et al. Histiocyte society review HLH-2004: diagnostic and therapeutic guidelines for Hemophagocytic Lymphohistiocytosis. Stockholm: Wiley-Liss; 2006.

19. Rahal AK, Fernandez J, Dakhil C. Undiagnosed systemic lupus erythematosus presenting as Hemophagocytic Lymphohistiocytosis. Case Rep Rheumatol. 2015:2015:748713.

20. Ideguchi H, Ohno S, Takase K, et al. Successful treatment of refractory lupusassociated haemophagocytic lymphohistiocytosis with infliximab. Rheumatology (Oxford). 2007;46:1621-2.

21. Keith MP, Pitchford C, Bernstein WB. Treatment of hemophagocytic lymphohistiocytosis with alemtuzumab in systemic lupus erythematosus. Clin Rheumatol. 2012;18:134-7.

22. Kumar B, Aleem S, Saleh H, Petts J, Ballas ZK. A personalized diagnostic and treatment approach for macrophage activation syndrome and secondary Hemophagocytic Lymphohistiocytosis in adults. J Clin Immunol. 2017;37: 638-43.

Ready to submit your research? Choose BMC and benefit from:

- fast, convenient online submission

- thorough peer review by experienced researchers in your field

- rapid publication on acceptance

- support for research data, including large and complex data types

- gold Open Access which fosters wider collaboration and increased citations

- maximum visibility for your research: over $100 \mathrm{M}$ website views per year

At BMC, research is always in progress.

Learn more biomedcentral.com/submissions 